New EE lab projects for non-EE majors: Fourier spectra of music and perception of the effects of student-built filters

Dr. Alexander Ganago, University of Michigan

Mr. Sudarshan Sivaramakrishnan, University of Michigan 


\title{
New lab projects for non-EE majors: Fourier spectra of music and perception of the effects of student-built filters
}

\begin{abstract}
Two laboratory projects for an introductory service course in Electrical Engineering (EE) for non-EE engineering majors relate the abstract concepts of Fourier spectra and transfer functions of filters to the students' favorite applications - creating and listening to the sounds of music. The projects include: (a) use of technology/software available on the Internet for the production of sounds and editing of audio files; (b) lab measurements of the waveforms and spectra of music, along with listening to the sounds; (c) soldering a multi-functional passive filter circuit and measurements of its transfer functions; (d) listening to the sounds of music without filters, through the software filters and through the real, soldered filters, and (e) comparison of the effects of these two types of filters on the sensory perception of the sounds of music. Here we explain the content of each project and its place in the sequence of rigorous measurements of standard signals; provide examples of lab data, and summarize the feedback from students and from the lab instructors in a large class.
\end{abstract}

\section{Introduction}

Among the challenges of teaching introductory courses in Electrical Engineering (EE) to non-EE engineering majors is the creation of laboratory projects that provide students an insightful hands-on experience, which would

$\checkmark$ Closely relate to theory and applications

$\checkmark$ Elucidate abstract EE concepts, and

$\checkmark$ Develop fundamental skills that can be readily applied to projects and environments that non-EE majors would encounter.

The constraints include:

$\checkmark$ Conceptual (students should be able to understand the engineering and physical principles in operation)

$\checkmark$ Logistic (the projects should be completed within a limited time in the lab)

$\checkmark$ Technical (the lab equipment and software should allow students to verify and alter their functionalities), and

$\checkmark$ Financial (the lab equipment and other required resources should be of low cost).

This report provides a general overview of a new set of EE lab projects for non-EE majors, with a special focus on the design and implementation of two new laboratory projects, which address the challenges and constraints listed above. Each of these two projects (Experiment 5-3 in the Spectra Lab and Experiment 6-4 in the Filters Lab) employs a multi-faceted, hands-on approach to learning that includes the creative use of technology/software available on the Internet for the production of sounds and the editing of audio files.

These projects were created and introduced in our teaching of a large introductory, service course in Electrical Engineering (EE) for non-EE students, mostly from our College of 
Engineering, in Fall 2012. Indeed, the overall course structure was changed at that time to incorporate more Lab experiments: during the semester, every student is required to do 8 Labs (instead of 6 in previous semesters). During the same semester, we designed and offered to the students many detailed online surveys that include multiple-choice and open-ended questions.

Our teaching goals include:

$\checkmark$ Focus on the key concepts and strategies transferable to many fields of engineering

$\checkmark$ Provide extensive lab experience in building/testing/studying a variety of circuits, which have been discussed in lectures

$\checkmark$ Engage students' interest via hands-on work with circuits for practical applications from the very beginning of the course.

Similar goals have been formulated and achieved by other instructors. For example, Sterian et al. (2008) included projects in their introductory circuits course that enrolls both EE and non-EE majors, with .

Our teaching strategy is to introduce realistic, practical applications in the lab projects (including the labs early in the semester) and make the concepts presented immediately relevant to the student audience. Our lab projects go beyond mere verification and reinforcement of the standard theory presented in the lectures. The practical applications that students learn in our labs help them transfer the skills and experience to future learning and work in their fields of major. In addition, we strive to develop projects that appeal to perception through a multiple senses in order to enhance learning. Lastly, we seek to create an engaging atmosphere in the lab that motivates students to apply what they learn in this EE course to their own interests, providing them a nice sampling of "cool" applications as a starting point.

Other authors also studied various aspects of teaching EE to EE and non-EE majors, including lab projects. For example, Sterian et al. ${ }^{1}$ developed a project-based approach to teaching introductory circuit labs with a special focus on intuitive understanding of simple circuits.

Unfortunately, their report does not provide information on the enrollment in their course, and includes only a cursory description of the surveys conducted in connection to the newly developed projects. Hajjar and Sobahi ${ }^{2}$ described the design of an introductory EE course for non-majors. From communication with universities, professors, research groups, and industry, they identified the need to present application-focused material, multidisciplinary systems with different types of sensors, and familiarity with software used outside the classroom (e.g. LabVIEW) but do not mention the laboratory component. Malik et al. ${ }^{3}$ examined the challenges that non-EE students encounter in an introductory EE course and emphasized the need for content that would be more engaging and relevant to students' interests. Northrup ${ }^{4}$ reported innovative lab experiments with many interesting and circuits of practical importance including temperature and light sensors, feedback control of motor speed, and music equalizers, but the level of difficulty is seemingly too high for non-EE majors.

Our approach is unique, because it engages students in many inter-related activities, including soldering a multifunctional filter circuit and testing its performance in the lab, using the software available on the Internet for conducting preliminary experiments, listening to the sounds of musical instruments and analyzing their waveforms and spectra, etc. It embraces theoretical 
learning with hands-on work, evokes sensory perception, and helps students relate their learning in the course to their favorite pastime of listening to music.

In this report we briefly outline the whole sequence of Lab experiments in the course, describe the Spectra Lab and the Filters Lab in more detail, summarize the statistics of students' answers to multiple-choice questions, and highlight the most interesting quotes from their open-ended comments.

Our lab development addresses ABET General Criteria for Baccalaureate Level Programs ${ }^{5}$, specifically regarding the section of Student Outcomes:

(3a) an ability to apply knowledge of mathematics, science, and engineering

(3d) an ability to function on multidisciplinary teams

(3e) an ability to identify, formulate, and solve engineering problems

(3i) a recognition of the need for, and an ability to engage in life-long learning

(3k) an ability to use the techniques, skills, and modern engineering tools necessary for engineering practice

\section{Background}

The course, for which we develop the labs, serves as an introduction to EE intended for engineering students from non-EE departments. The goals of this course include:

$\checkmark$ Help non-EE students learn the key EE concepts and skills they can use in projects across many fields of engineering

$\checkmark$ Highlighting practical applications in which EE is used, and

$\checkmark$ Providing hands-on, insightful, and enjoyable lab experience.

\section{Student audience}

In the Fall 2012 semester, enrollment was 156 students, including 90 juniors, 64 seniors, one sophomore, and one graduate student. As for their fields of study, the primary audience was composed of Mechanical, Aerospace, and Nuclear engineering students, as shown in Figure 0. 


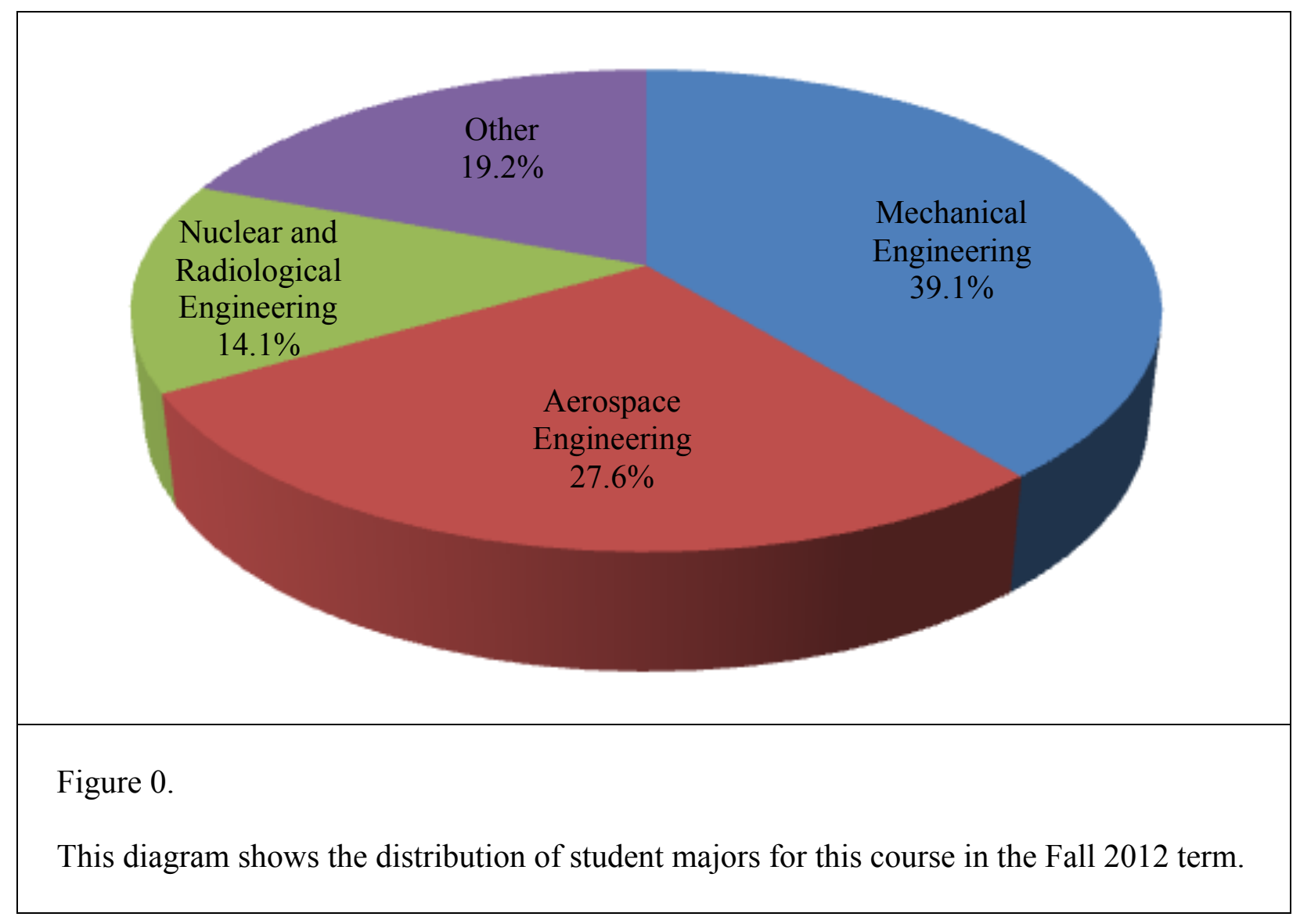

\section{*verall structure and content of the labs}

For Fall 2012 the number of lab projects was increased from 6 to 8 per semester, all required of every student. Each lab project has been designed to be completed within 2 hours of the scheduled time; students work in the lab in teams of two but submit their lab reports individually. Each of the key concepts was first introduced in the lecture, then practiced in homework and discussion sections, and eventually reinforced in the lab experiments. Before offering the new course structure in the Fall 2012 (156 students enrolled), many innovations were tested in the Spring 2012 (34 students enrolled).

All lab projects are listed below. Each of the first 6 Lab projects included several Lab Experiments in modular form; for example, Lab 1 includes 4 independent Lab Experiments, or modules numbered 1-1 through 1-4. This structure allows the instructor to skip or swap individual modules in order to tailor the content of the labs to the overall course syllabus, time constraints, student backgrounds, etc. Each Lab Experiment, or module, includes: Goal and overview, Introduction, Pre-Lab, In-Lab, and Post-Lab.

Lab 1. Tutorial: Start using the Lab resources for learning 1-1. Use the Digital Multimeter (DMM) to measure voltages, currents, and resistances 
1-2. Use the power supply with built-in voltmeter and ammeter to study how Ohm's law holds and fails

1-3. Use the Virtual Instrument "Lab_1_IV Diode Plot" to obtain the Volt-Amp characteristic of an Light-Emitting Diode

1-4. Use the function generator and oscilloscope to produce sine waves and measure their waveforms and spectra

Lab 2. Sensor and Switch: Build your own control circuit

2-1. Verify KCL and KVL; Measure the power in circuits with two lamps connected in parallel and in series

2-2. Photocell as a Sensor: Resistances and Voltages

2-3. MOSFET as an Electronic Switch

2-4. Build Light-Activated Control Circuits with a Photocell as Light Sensor and a MOSFET as Electronic Switch, which automatically turn On/Off the Actuator (lamp or motor)

Lab 3. Waveforms: Time-dependent voltages in and around you

3-1. Explore a variety of waveforms; Measure Rise/Fall Times

3-2. Telephone Dialing Tones

3-3. Build an RC circuit and measure its exponential responses

3-4. Build an LC circuit and measure its oscillatory response

Lab 4. Transients: They limit the speed of your computer

4-1. Rise/Fall Time and time constant $\tau$ of RC circuit response

4-2. Are there oscillations in your RLC circuit?

4-3. The Photonic Link: Unexpected Transient Responses

4-4. The Pulse Counter: Analog Behavior of Digital circuits

Lab 5. Spectra: Frequency composition of familiar signals

5-1. Spectra of standard signals

5-2. Use the Fourier Synthesizer to build the Square and Ramp waveforms from their sinusoidal components

5-3. Spectra of pure tones, overtones, and musical instruments

Lab 6. Filters and Soldering: One circuit serves as many filters

6-1. Solder your own circuit and prove that it is built correctly

6-2. Low-Pass filter

6-3. High-Pass filter

6-4. Resonance in LC and RLC circuits

6-5. How the Low-Pass and High-Pass filters affect the sounds of music

Lab 7. Op Amps: Build and study amplifiers and other circuits

Lab 8. Temperature controllers: Build, study, and compare Analog/Digital and

Programmable Devices

Our intent is to help students: 
- First understand the material in each module independently, and then

- Establish connections between modules.

We assume that, in this way, students would appreciate how the various concepts are connected and interrelated:

- How a given Lab reinforces and extends upon their learning in the previous Labs, and

- How each Lab becomes a part of the foundation for the learning in the subsequent Labs.

After the students completed each project, we offered them a survey to find out

- What parts of the lab were most interesting/valuable, and what remained unclear

- How the students understood the connections between the lab experiments, and

- How effective lab learning was in the overall learning of EE.

\section{* Description of lab projects}

The Spectra Lab and Filters Lab are presented in further detail below. In all experiments, we use Agilent 33220A function generator and DSO-X-2012A oscilloscope; many other instruments would yield similar results.

The Spectra Lab has been divided into three Experiments, or modules (see above).

In Experiment 5-1, students use the function generator to produce standard signals (sine, square, and ramp/sawtooth waves) and the oscilloscope to observe the waveforms and acquire their FastFourier Transform (FFT) spectra. The Pre-Lab assignment asks students to read through an introduction to the Fourier series for these standard signals and compare theoretical calculations to sample data provided in the lab manual. In the lab, students work with the same and other signals, measure and record their spectra. The Post-Lab assignment asks students to compare their own lab data with theory, as well as review their measurements of the signal-to-noise ratio in FFT spectra and of the total harmonic distortion for a clipped sine wave. This module helps students appreciate the relevant functionalities of the instruments and understand the spectra of standard waveforms; it is heavily focused on quantitative comparison between measured frequencies and amplitudes to theoretical predictions, and serves as an important precursor to the subsequent modules.

In Experiment 5-2, students make use of a LabVIEW® program, or Virtual Instrument (VI) called Fourier Synthesizer, which we developed several years ago and reported elsewhere. Its front panel is shown in Figure 1, which is taken from the lab manual. 


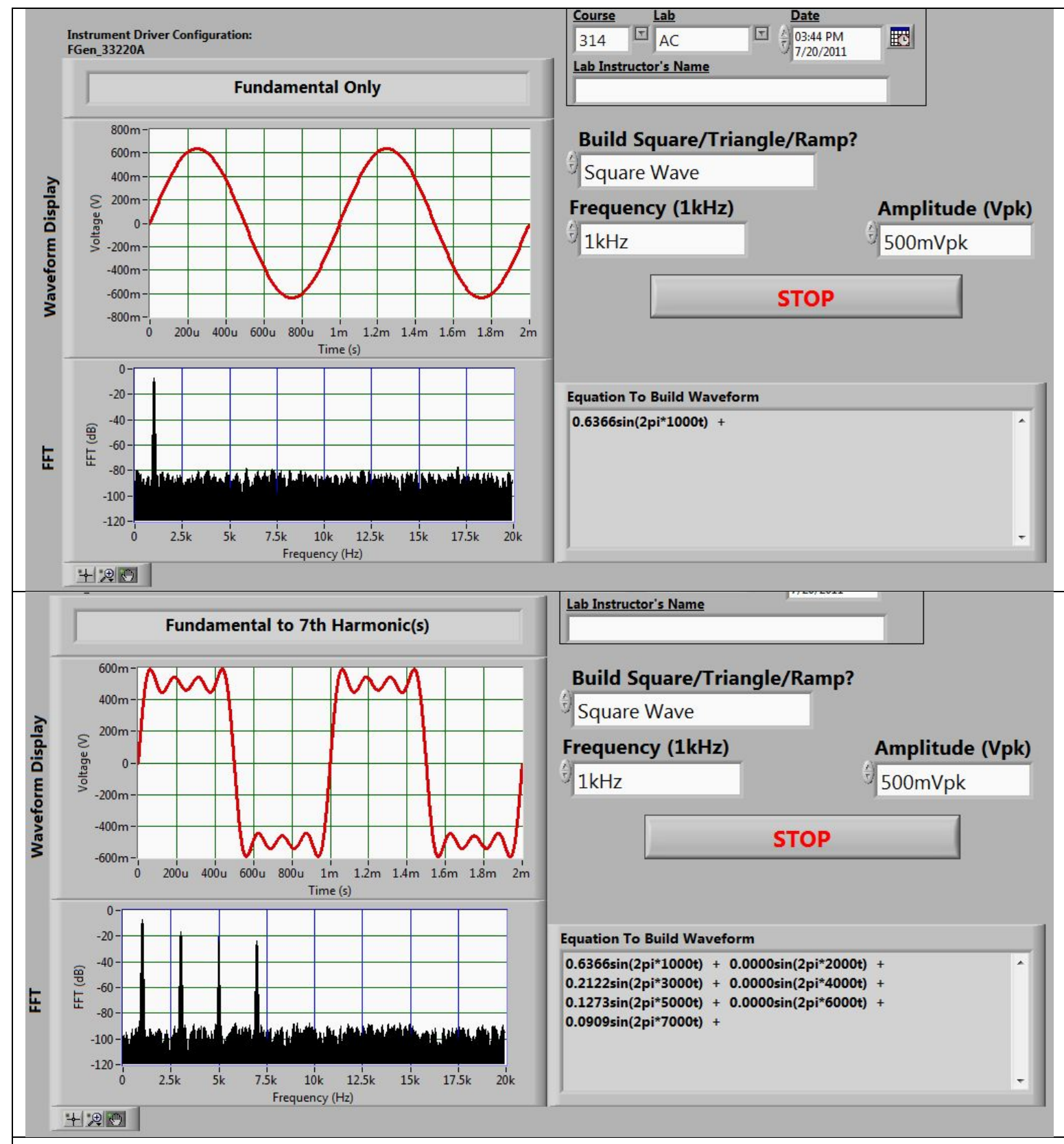

Figure 1 (from the lab manual).

The front panel of Fourier Synthesizer.vi includes the fields to be filled by the user (choice of the waveform, frequency, and amplitude) and the windows where the computer builds the desired signal (the waveform, its FFT spectrum, and the algebraic expression used to calculate the sum of sinusoidal spectral components).

The computer controlled with this VI builds the chosen waveform step-by-step as a sum of its harmonic components (see Figure 1 and its legend for details); at each step it produces the resulting waveform (partial sum of harmonics), displays it on the front panel of the VI, and sends 
it to the function generator whose output can drive a speaker so that the students can listen to the sounds and appreciate their evolution when every new harmonic component is added to the sum. The lab manual emphasizes that the fundamental acts as a pure musical tone, and the harmonics act as overtones. In summary, the use of this VI provides a comprehensive learning experience: the abstract characteristics of waveforms (analytical expression, spectrum, etc.) are combined and enhanced with the sensory perception of sounds, to which the students can easily relate.

We focus our explanation on the next module of the Spectra Lab, Experiment 5-3, because it implements our new way to help students relate highly abstract theoretical concepts to their familiar activity of listening to music. This Experiment extends the explorations of waveforms, sounds, and spectra to the signals produced by familiar musical instruments. Thereby it takes the students above and beyond the conventional scope of introductory courses limited to simple spectra of standard waveforms (which we covered in Experiment 5-1). It also builds on their learning in Experiment 5-2, which fosters students' intuition and appreciation of how waveforms are created out of sine waves. Experiment 5-3 makes use of a Virtual Keyboard, which is available as a freeware on the Internet (shown in Figure 2), in order to mimic the sounds of several musical instruments.

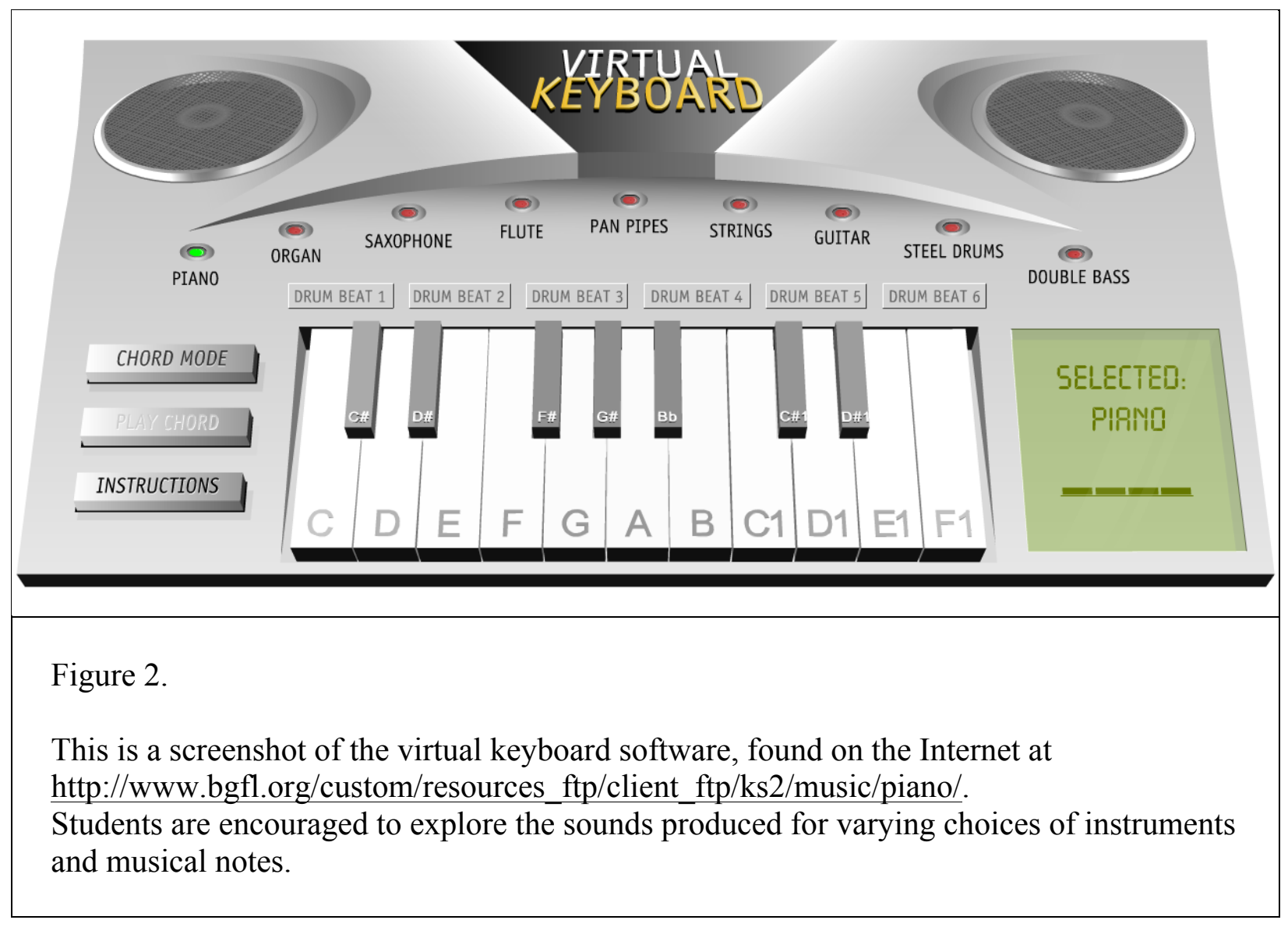

In the Pre-Lab for Experiment 5-3, we encourage students to familiarize themselves with the software (at home or in a computer lab on campus), listen to the sounds it produces, and to make predictions about the spectral properties of some notes "played" by several instruments. For 
example, they are asked which of the instruments playing a single note create the sound that have only few harmonics - and which ones produce the sounds with many harmonics. We emphasize that it is a preliminary exploration, and their answers will not be graded for correctness.

In the lab, students run the same Virtual Keyboard software on the computer on their lab desk, connect the audio output of the computer to the oscilloscope in order to measure the waveform and spectrum, and at the same time connect it to the speakers at the lab station in order to hear the sounds. Students then apply the skills they acquired from the first two modules to analyze the waveforms and spectra of the various musical instruments, and they compare their measurements and visual observations with what they hear as well as their Pre-Lab predictions. An example is shown in Figure 3.

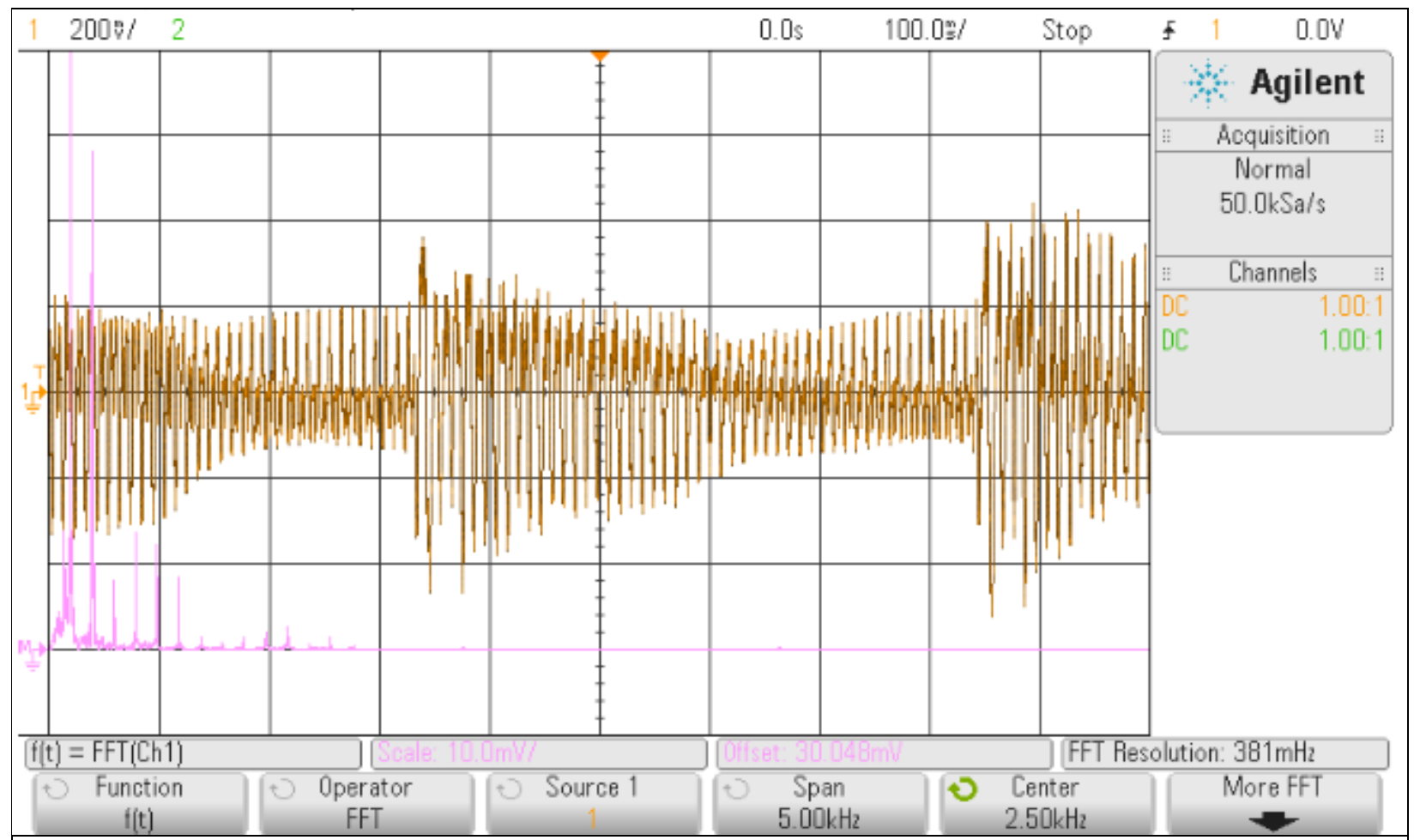

Figure 3.

This is a screenshot of the oscilloscope screen, capturing the waveform and spectra associated with the note $G$ played on the virtual keyboard with the instrument setting as Double Bass. The orange trace shows the waveform (near the center of the screen), and the light purple trace shows the FFT spectrum (in the lower left corner of the screen).

In addition to the required work outline above, Experiment 5-3 offers a fun, optional component, in which students connect their mp3 players to the oscilloscope and speaker and then observe more complicated waveforms and spectra of their favorite songs. This provides an opportunity for open-ended exploration driven by the students' own curiosity and interest. 
In our experience of teaching this Lab, the 2-hour scheduled time was sufficient for all student teams to complete all three modules. The stronger teams finished the required assignments earlier thus they had more time for fun listening to their favorite music and exploring its spectra.

The Filters Lab has been divided into five modules. The first module, Experiment 6-1 requires that each team of 2 students solder their own circuit (Figure 4), to be used in the further Experiments 6-2 through 6-5.

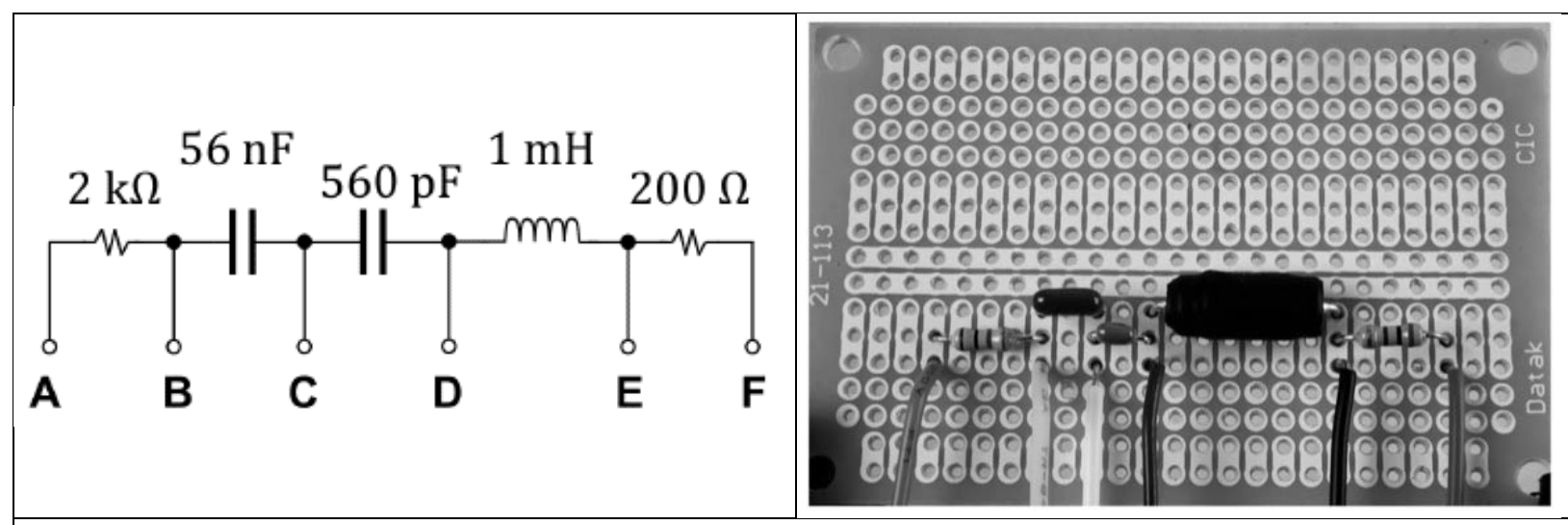

Figure 4 (from the lab manual).

The above circuit diagram is provided to students, and they are asked to build a soldered filter circuit on a prototyping board, as shown in the photograph.

We jokingly call this circuit "a Swiss Army knife circuit" because it can serve as many filters, depending on how you connect the input and the output: Low-Pass and High-Pass with various cutoff frequencies (Experiments 6-2 and 6-3), Band-Pass and Band-Reject with various Q-factors (measurements of these two filters are not included in this Lab); it also allows students to observe the buildup and decay of the resonant response in Experiment 6-4.

The lab manual for Experiment 6-1 includes a brief introduction to soldering, which covers the underlying physical principles, a safety review, and links to videos demonstrating proper soldering techniques. In the Pre-Lab assignment, students are asked to draft a layout of components on a diagram of the prototyping board and answer questions reviewing the soldering process. In the lab, they are given step-by-step instructions (accompanied with numerous photographs) to solder their filter circuit shown in Figure 4. Then, they perform a set of basic measurements to verify that their soldered connections are adequate and that they have built their circuits correctly. This soldered filter circuit is then used for all subsequent modules.

In the second module, Experiment 6-2, students use their soldered circuit as a Low-Pass RC filter. In the Pre-Lab assignment, students are asked to draw the correct connections of the circuit to the function generator and oscilloscope in preparation for the lab. In addition, students make plots of the theoretical transfer function magnitude. In the lab, each student team begins by connecting its function generator and oscilloscope to their soldered circuit as a Low-Pass 
filter, according to the given specifications. Students perform manual measurements at a few frequencies to prove that it acts as a Low-Pass filter and then make use of a Virtual Instrument called Bode plot (see Figure 5) to characterize the Low-Pass filter and understand its cut-off frequency and transfer function, drawing comparisons to theoretical predictions from the PreLab. In the Post-Lab, students are asked to compare their lab data with theory.

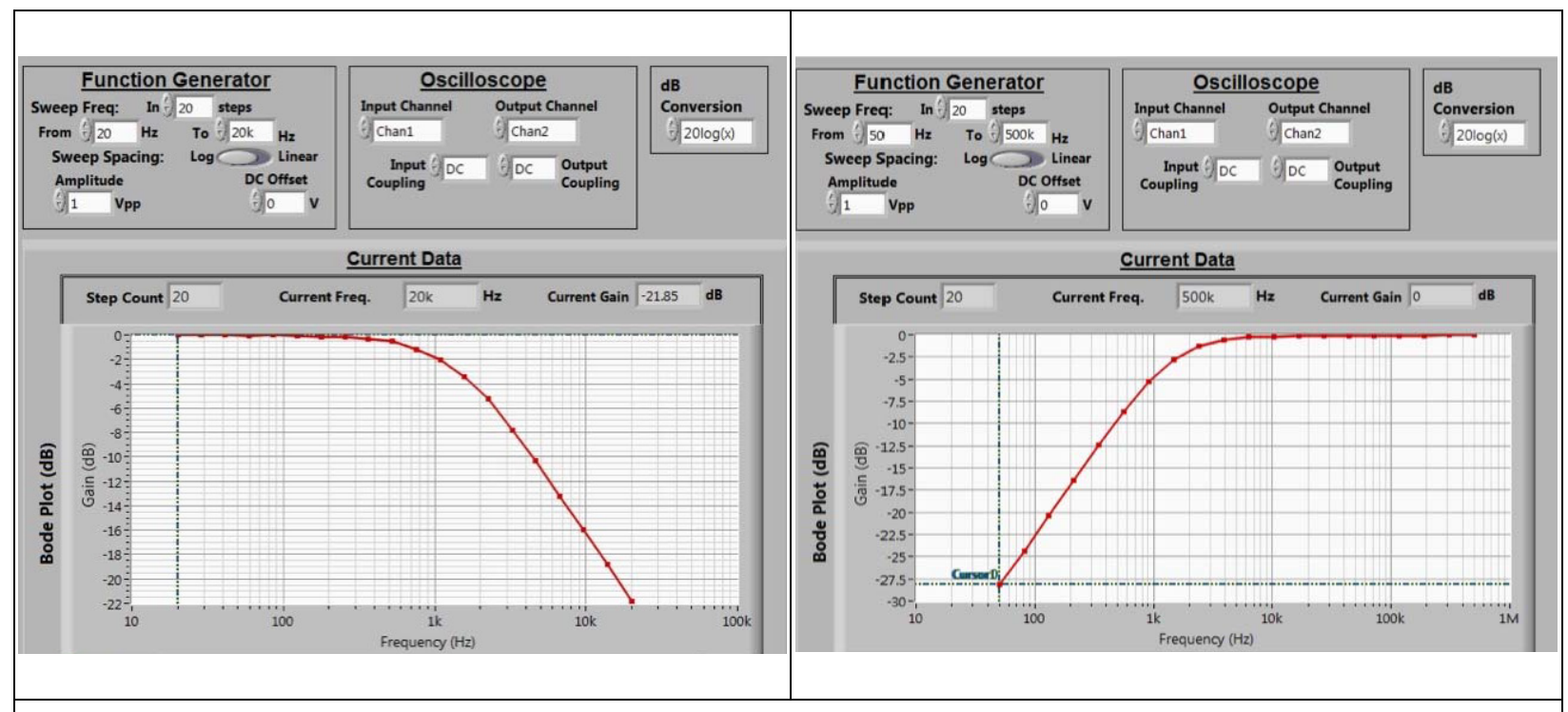

Figure 5.

These screenshots of the front panel of the Bode plot VI show typical lab data of transfer function magnitudes obtained on the soldered circuit (Figure 4) used as Low-Pass and High-Pass RC filters (left and right panels, respectively). Note that the data embrace the frequencies of the human hearing range, because these filters are specifically designed to serve as audio filters for music, as detailed in Experiment 6-5.

The third module, Experiment 6-3, is very similar to the second module, except that it makes use of the soldered filter circuit as a High-Pass RC filter.

In the next module, Experiment 6-4, students use the function generator in its Burst mode to produce 30 periods of the sine wave at the resonant frequency of their soldered circuit, and observed the gradual buildup and decay of the resonant response. When they connected their circuit as LC, the resonance was strongly pronounced and took more than a half of the 30 periods to reach the sinusoidal steady state; when the circuit was connected as RLC, the losses made the resonant response smaller, reaching the steady state faster, and decaying within a shorter time. Since the buildup and decay of the resonant response cannot be explained with phasors, and we do not cover Laplace transform in this course, the Pre-Lab and Post-Lab analysis was limited to calculations of the expected resonant frequency and semi-quantitative description of the data.

Again, we focus on our innovation - the fifth module for the Filters Lab, Experiment 6-5, which helps the students relate the abstract concept of transfer function magnitude to their sensory 
perception of music. We offer the students a brief recording, which they listen in three ways: without filtering, through a Low-Pass filter, and through a High-Pass filter. Then, as an option, they can apply the same filters to different music recordings. As the standard sample of music we chose a 10-seconds initial fragment of the popular Beatles' song Help! because it contains sounds from three distinct sources in three different frequency ranges (Figure 6): a bass guitar at the lowest frequencies, vocals in the mid-range, and cymbals at the highest frequencies.

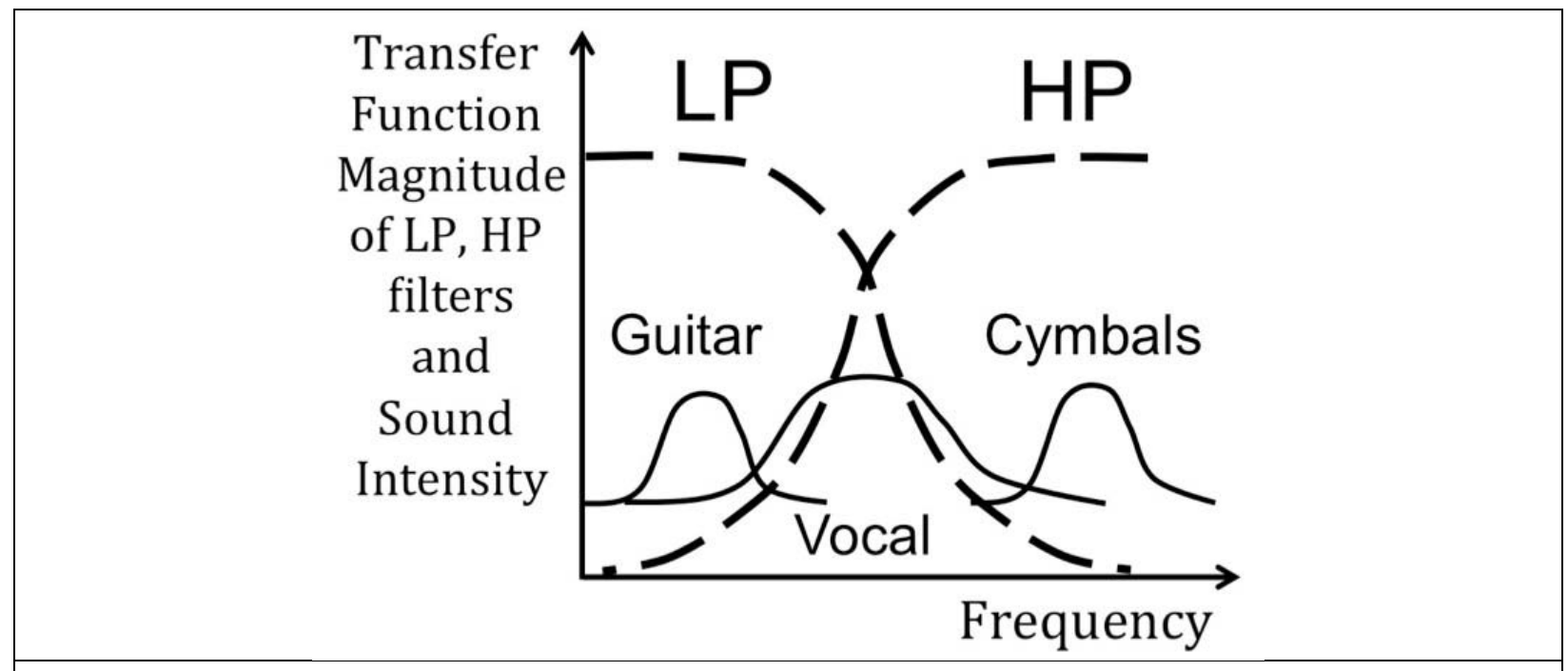

Figure 6.

This sketch approximately indicates the three separate spectral regions, which correspond to the sounds of the bass guitar, the vocals, and the cymbals/percussion in the first 10 seconds of the Beatles' song Help!, relative to the transfer function magnitudes of the Low-Pass and High-Pass filters soldered by the students.

Specifically for the teaching purpose, we reduced the stereo sounds of the Beatles' recording to a much less enjoyable monophonic track, 10 seconds long, which students used in the Pre-Lab and in the lab. We guided our design of the Low-Pass and High-Pass filters for this Lab to the spectrum of this monophonic track and chose the cutoff frequency of $1421 \mathrm{~Hz}$ because it ensured the selective effects on the sounds, as shown in Figure 6. Our goal was to ensure that the students could easily perceive the effects of Low-Pass and High-Pass filters.

In the Pre-Lab, students make use of the open-source software Audacity to apply Low-Pass and High-Pass filters created with this software to the excerpt of music, and they record their qualitative observations (Figure 7). This software allows the user to choose the type of filter, its cutoff frequency, and other parameters. In the Pre-Lab assignment, students used the filter parameters matching the components of the filter circuit that they would later solder in the lab.

In the lab, students listened to the same music again, this time using their filter circuit connected either as High-Pass or Low-Pass. Again, they record their qualitative observations (Figure 7). Students were also encouraged to include additional observations and comments. In the Post- 
Lab assignment, students draw comparisons between the perceived functionality of the "software" filters from the Pre-Lab and the equivalent "hardware" filters soldered in the lab.

\begin{tabular}{|c|c|c|c|}
\hline Do you hear these? & No filter & Low-Pass filter & High-Pass filter \\
\hline Bass guitar & & & \\
\hline Vocals & & & \\
\hline Cymbals/Percussion & & & \\
\hline
\end{tabular}

Figure 7.

The students recorded their observations in the Pre-Lab and in the lab in this table.

As a fun, optional part of this Experiment, students are encouraged to apply the soldered-circuit filters to their favorite music. They bring their $\mathrm{mp} 3$ players to the lab, connect the player's output as they used to connect their computer's output, and observe the effects of filtering on various genres of music and types of sounds found in their favorite songs. Again, this provides an opportunity for open-ended exploration for each student, driven by his or her own interest. It also allows students to enrich their learning and pose questions in ways that were not considered when developing the lab.

Our teaching experience in Fall 2012 showed that all 5 modules of the Filter Lab do not fit into the scheduled 2-hour time for in-lab work. Therefore we made the In-Lab components of Experiments 6-3 (High-Pass filter) optional. The students were required to do the Pre-Lab calculations, and were given sample lab data in order to complete their Post-Lab comparison with theory.

\section{* Summary of student feedback}

Striving to evaluate student learning in the lab, we have designed and implemented a set of comprehensive surveys with multiple-choice and open-ended questions, combining quantitative and qualitative methods of analysis. We used a professional version of SurveyMonkey® that collects responses on one web site and collects the students' personal information on another web site; thus we maintained students' anonymity and awarded a small amount of extra credit for completion of each survey. Here we provide summaries of survey results for the Spectra and Filters Labs.

$\underline{\text { Statistics of the student feedback for Spectra Lab }}$

80-90 students participated in the survey on the Spectra Lab. Figure 8 presents the statistics of their responses to multiple-choice questions. 


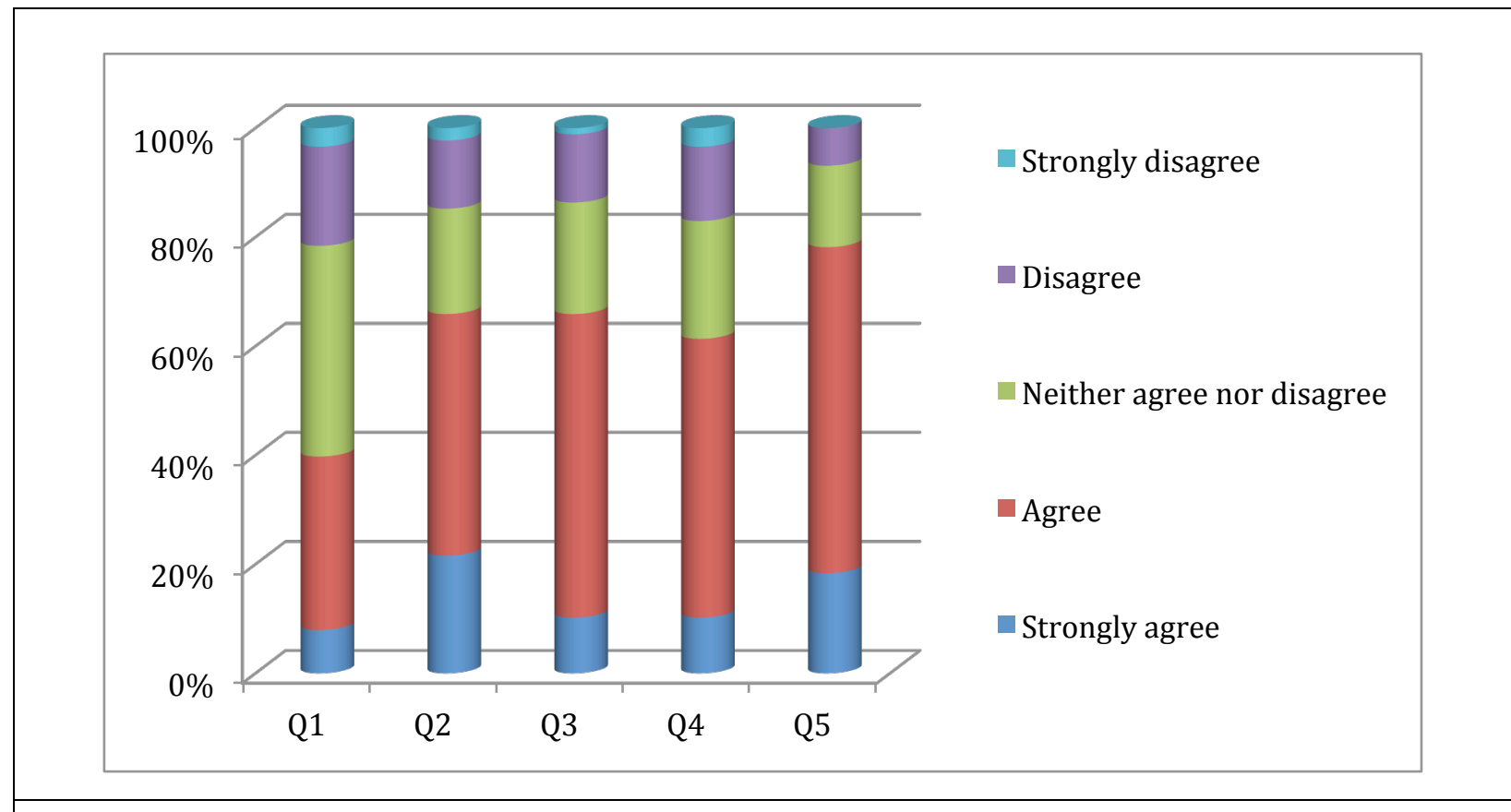

Figure 8.

The statistics of students' responses to the following statements regarding the Spectra Lab:

Q1: "I feel that my learning in Lab 5 is valuable for what I do outside this course"

Q2: "I had enough time in the lab to think about what I was doing"

Q3: "I understood each step of the In-Lab work"

Q4: "I feel that my learning in previous Labs (1-4) helped me in Lab 5"

Q5: "Lab 5 has been interesting"

One of the main goals of this course for non-EE engineering majors is to foster the students' understanding of how the EE concepts are applied to their fields of major. Figure 8, Q1 indicates that $\sim 40 \%$ "Strongly agree" and "Agree" $(\mathrm{SA}+\mathrm{A})$ that this goal was achieved in the Spectra lab. Here and below, the numbers quoted in the text belong to the sum $(\mathrm{SA}+\mathrm{A})$.

Another key element for success in any Lab experiment is to provide students with enough time to think about what they are doing in the lab, without feeling rushed to complete the required steps at any cost. Figure 8, Q2 and Q3, indicates that this goal was achieved by $\sim 66 \%$ of the respondents.

Our more comprehensive goal was to create a sequence of Lab projects that gradually builds student understanding and skills. Figure $8, \mathrm{Q} 4$ indicates that $>60 \%$ of our students this progression of learning.

Last but not least, we strive to create an enjoyable learning environment. $62.5 \%$ of the respondents agreed or strongly agreed with the statement "I enjoyed doing In-Lab 5" (we do not 
show this data in Figure 8). Student interest in the course material is important for us. Figure 8, Q5 indicates that $>70 \%$ of the respondents found the Spectra Lab interesting.

The open-ended survey questions provide a deeper insight into student learning. We review them in a later subsection.

Statistics of the student feedback for Filter Lab

About 90 students submitted their responses to our survey on the Filter Lab: see Figure 9.

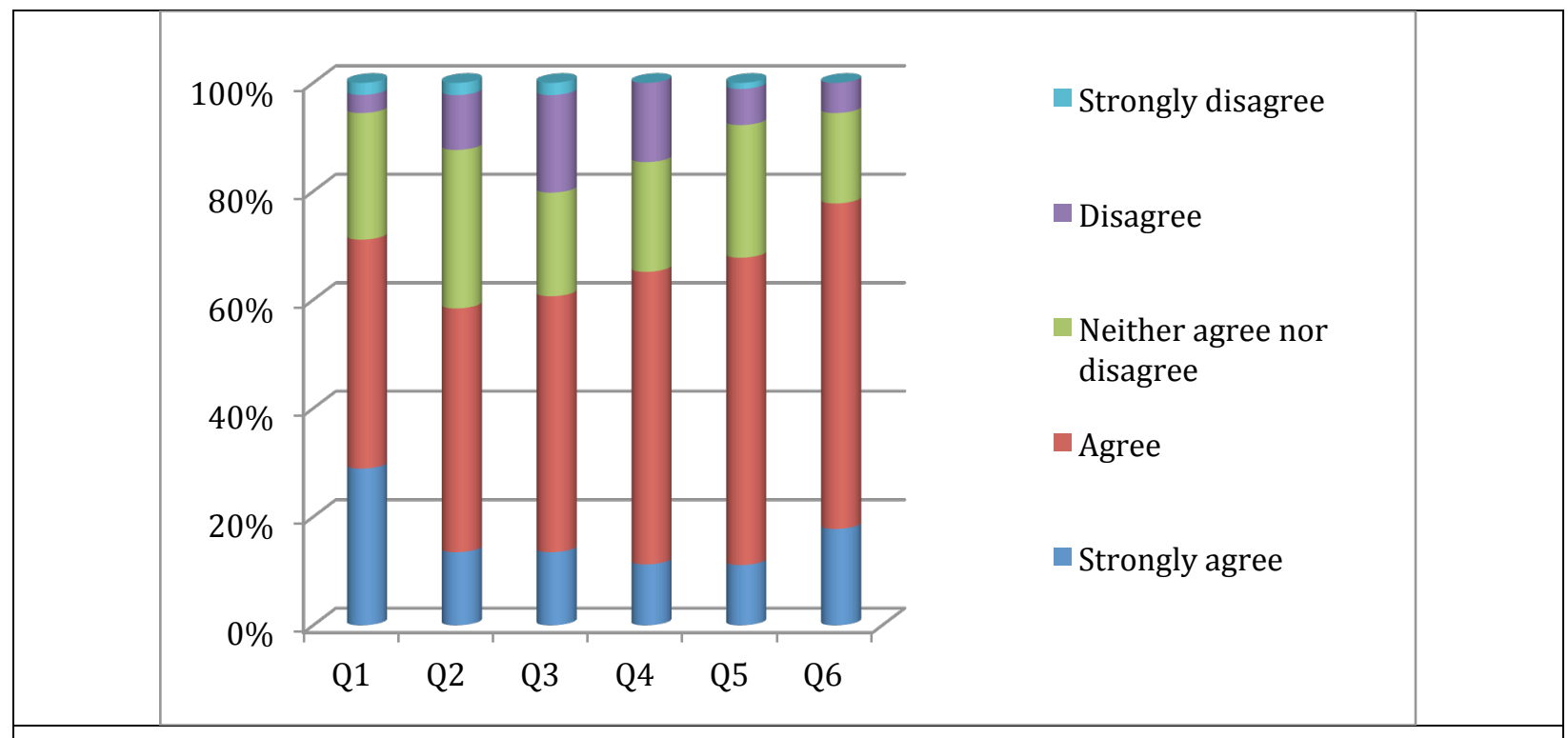

Figure 9.

The statistics of students' responses to the following statements regarding the Filter Lab:

Q1: "Listening to music helped me understand how filters work"

Q2: "I feel that my learning in Lab 6 is valuable for what I do outside this course"

Q3: "I had enough time to think about what I was doing"

Q4: "I understood each step of the In-Lab work"

Q5: "I feel that my learning in previous Labs (1-5) helped me in Lab 6"

Q6: "Lab 6 has been interesting"

As outlined above, we use a variety of approaches to foster students' understanding of filters. Figure 9, Q1 indicates that listening to music helped $\sim 70 \%$ of the respondents to develop this understanding.

Figure 9, Q2 indicates that $\sim 60 \%$ of students relate their learning of filters to what they do outside this course. Compared to Figure 8, Q1 for the Spectra Lab, this number is much higher. 
Again, we asked the questions on timing of the in-lab work. Figure 9, Q3 and Q4, indicate that $60 \%$ of respondents agreed that the scheduled time was sufficient for thinking and understanding of the required procedures. This is similar to Figure 8, Q2 and Q3, regarding the Spectra Lab.

Nearly $70 \%$ of the respondents felt that their learning in earlier Labs helped them in the Filter Lab (Figure 9, Q5). This is slightly higher than what Figure 8, Q4 shows for the Spectra Lab.

The student interest in doing the Filter Lab (Figure 9, Q6) is high, similar to the responses regarding the Spectra Lab (Figure 8, Q5).

\section{* Review of the open-ended questions}

Our first question regarding the Spectra Lab was "What is the most memorable thing about Lab 5?" Of the 91 responses, more than half of the students pointed specifically to content from Experiment 5-3. A few representative quotes are provided below:

- "Being able to see the different frequencies and waveforms of the various instruments, it was actually quite interesting to see what we are actually hearing"

- "The most memorable thing was DEFINTELY the experiment about virtual keyboard and the music!! :)"

- "the most memorable thing about the lab was that musical instruments have very different waveforms and spectra than pure tones."

Next, we asked our students "What do you think is the most important thing that you learned in Lab 5?" In general, students referred to obtaining/reading spectra on the oscilloscope, spectral composition and synthesis of various sounds/waveforms, and the process of adding harmonics to synthesize waveforms. Here are some answers relating to the new Lab experiments:

- "Fourier approximations finally make sense"

- "The most important thing I learned in lab 5 was Fourier's theorem. I have seen this in other classes, and now that I have seen a practical application, the theorem is more clear. This theorem is very important for my other classes in my major."

We then asked students, "How can you apply your Lab 5 experience to your endeavors outside the course?" Many students reported that they could use the understanding of spectra, bandwidth of circuitry, and Fourier's Theorem gained from this experience in their future work. However, a number of students felt they could not readily apply the lab material to their field of study, but more perhaps for musical hobbies. This implies that further work needs to be done to establish the application of this knowledge in various engineering fields. Here are some hesitations:

- "If I work in the audio industry it would be vital, can't think of many reasons outside of that."

- "I am not sure yet. I would have to spend more time on the topic to feel comfortable with the material enough to apply it." 
To assess how the lab interrelates with the rest of the course material, we asked "How well did this Lab fit in with the other parts of the course?" The students' comments were very positive:

- "This lab is what I expected out of most of this course; it was fun, related to real devices/things we use every day, and made sense. This was the best lab so far."

- "It fit in well. It gave hands on understanding of topics covered in lecture."

- "It allowed us to use the skills we have learned inside of the lab and apply them to things that we encounter every day, like musical instruments. I was able to use my in lab skills to observe things I couldn't normally see."

For the Filter Lab, we first asked, "What is the most memorable ... interesting ... insightful thing about Lab 6?" Of the 97 responses, $\sim 2 / 3$ of the students reported the soldering experience; also, $\sim 1 / 2$ of the students mentioned applying filters to music and observing the effects. Overall, responses expressed enjoyment and enthusiasm, as well as a sense of accomplishment for having built a complete circuit with a desired functionality that can be readily tested and observed in lab. Representative quotes are given below:

- "I really enjoyed soldering my own circuit and seeing/hearing first hand how the high/low pass filters work was great."

- "It was interesting to hear how filters work instead of just trying to understand how they work without any evidence."

- "I liked listening to the Beatles song to see how low pass and high pass filters affected the song. It was a much more memorable and interesting way to sense the frequency."

We also asked the students "How do you see the connection between Lab 5 = Spectra and Lab 6 = Filters and Soldering?" Most students appreciated a certain connection between the labs; some included specific statements like the following:

- "You have to know about spectra in order to see the effect of filters"

- "The filters in lab 6 alter the spectra observed in lab 5."

- "Seeing which spectra are dominant in various instruments [in Lab 5] helped me see how the instruments in the song clip would get cut out with the two filters [in Lab 6]."

Our other questions encourage students to express their opinion on the music part of the Filter Lab, its value for learning, and connection to their studies and work. The answers were positive, interesting, and sometimes unexpected, for example:

- "This part was very interesting. This was the part that helped me understand the physical aspect of harmonics of waves and their frequencies. The sounds of music was not exactly relevant to my interests, however it did help me understand (just a bit more) the construction of wave functions that apply to quantum mechanics (Nuclear Engineering)."

- "It is VERY cool certainly! I find it amazing that I now have the knowledge to build a music filter, and it is VERY much relevant to the field of study I am interested in. It has helped me to see the effects of what we do in theory on a VERY COOL real life application :)"

- "More coursework should be like this. Engagement = learning." 
We finally asked, "Has this lab strengthened your understanding of the connection between theory (both the physical concepts and mathematical formulations) and experiment (what you do, see, hear, and observe in the lab)? If yes, how? If no, then what do you think is lacking?" Nearly all student answers responded positively to this question. Below are some representative responses:

- "Yes. Before, I only understood it through the formulations and looking at the circuits on paper. Having to build the certain filters and then hearing the sounds and lack of sounds helped put a physical result with what I had thought I knew. It helped legitimize the theory."

- "Yes, the use of the music signal was especially illustrative."

- "Yes, being able to see something happen in electrical engineering is rare, let alone being able to build something. If we could build something in every lab, this would be the best lab ever (as long as we don't have to print a lot of graphs)."

\section{Discussion}

The results of our teaching and surveys indicate the success of our strategy for Lab development and fostering student learning, engagement, and enthusiasm about the course material. They are matching the ABET General Criteria for Baccalaureate Level Programs, specifically regarding the section of Student Outcomes ${ }^{5}$.

In particular:

(3a) Ability to apply knowledge of mathematics, science, and engineering;

(3k) Ability to use the techniques, skills, and modern engineering tools necessary for engineering practice.

In these Labs, students apply their knowledge of Fourier spectra and filters' transfer functions to their everyday activity of creating and listening to music. They develop valuable practical skills of soldering and using modern oscilloscope to analyze the waveforms and spectra of signals. Eventually, students appreciate the connections between this learning and engineering practice in their fields of major.

(3d) Ability to function on multidisciplinary teams.

In the Labs of this course for non-EE majors, students function in multi-disciplinary environment: the two students in each in-lab team may be of different majors, and the EE Lab instructor definitely has a major distinct of any student in the lab. In our surveys (reported elsewhere) we found that students develop valuable skills in this course and feel comfortable in multi-disciplinary teams and working environment.

(3e) Ability to identify, formulate, and solve engineering problems

The Lab projects described in this report train students to identify engineering problems in the real-world environment (for example, while listening to music, ask what is the 
spectrum of these signals, and how would filters affect it), to formulate these problems in professional terms (units of frequency for signals, the types of filters and their parameters such as the cutoff frequency), and to solve problems as engineers (build the filter of a particular type and with the desired parameters, measure the waveforms and spectra, etc.). Interestingly, many students initially overlooked or at least undervalued the fact that music and other arts have very rich engineering applications; thus the goal of our work is to broaden students' perspectives and inspire them to be open-minded as they look for innovative ways to apply their engineering skills.

$$
\text { Recognition of the need for, and an ability to engage in life-long learning }
$$

These lab projects help students recognize that the concepts and skills they learn in EE class are applicable to their professional and leisure activities, and appreciate the benefit of life-long learning that may be applicable to their activities in the future.

In addition, it is to be noted that although students do not participate greatly in the design of the systems and circuits in the lab projects, they gain experience in building according to someone else's design (namely us, the lab developers). Insofar as addressing ABET criteria (3e), our set of lab projects provide a starting platform for students to apply EE concepts to search for new engineering solutions.

\section{References}

1. Sterian, A., Adamczyk, B., and Rahman, M. M. A. A project-based approach to teaching introductory Circuit Analysis (2008). Paper S1F-3 presented at the $38^{\text {th }}$ ASEE/IEEE Frontiers in Education Conference, Saratoga Springs, NY

2. Hajjar, A. F., and Sobahi, N. M. Basic electrical engineering for non-majors: course design and implementation . (2011) Global Journal for Engineering Education

3. Malik, Q., Mishra, P., Shanblatt, M. Identifying Learning Barriers for Non-major Engineering Students in Electrical Engineering Courses (2008). Proceedings of the 2008 ASEE North Central Section Conference

4. Northrup, S. G. Innovative Lab Experiences for Introductory Electrical Engineering Students (2009). Paper M4H-1 presented at the $39^{\text {th }}$ ASEE/IEEE Frontiers in Education Conference, San Antonio, TX

5. ABET criteria (http://www.abet.org/DisplayTemplates/DocsHandbook.aspx?id=3143) 